\title{
Identification of the Gene Regulatory Region in Human rasgrp2 Gene in Vascular Endothelial Cells
}

\author{
Kentaro Nagamine, * Akira Matsuda, and Takamitsu Hori \\ Laboratory of Biochemistry, Hiroshima International University; 5-1-1 Hirokoshingai, Kure, Hiroshima 737-0112, Japan. \\ Received February 26, 2010; accepted April 5, 2010
}

\begin{abstract}
Ras guanyl nucleotide-releasing protein 2 (RASGRP2) is a calcium- and diacylglycerol-responsive guanine nucleotide exchange factor. Previously, we reported that XRASGRP2, a homolog of human RASGRP2, is expressed in the vascular region of the Xenopus embryo during embryogenesis. Here, we report the regulatory mechanisms of human rasgrp 2 gene expression in vascular endothelial cells. Although three alternative splicing variants, differing in the first exon of $5^{\prime}$-untranslated region ( $5^{\prime}$-UTR), have been found for the human rasgrp 2 gene, reverse transcription-polymerase chain reaction (RT-PCR) showed that the cDNA containing the distal first exon (D1E) was expressed only in human umbilical artery endothelial cells. We analyzed the $5^{\prime}$-flanking region of the human rasgrp2 gene by a luciferase assay, which revealed that not only a promoter but also silencer regions were present upstream of D1E, suggesting that rasgrp 2 expression is controlled by a combination of transcriptional promotion and repression. Gel super shift assay demonstrated that OCT1/POU2F1 bound to the silencer region. These findings may help furthering our understanding of vasculogenesis and/or angiogenesis in vascular endothelial cells.
\end{abstract}

Key words vascular endothelial cell; angiogenesis; promoter; calcium and diacylglycerol guanine nucleotide exchange factor I; Rap; Oct1

The ras guanyl nucleotide-releasing proteins (RASGRPs) are a family of calcium- and diacylglycerol (DAG)-responsive guanine exchange factors, and permit activation of Ras and Rap. ${ }^{1,2)}$ The family members share the C1 DAG-binding domain, calcium-binding EF-hand domain, and guaninenucleotide exchange factor domain.

We recently constructed aggregates formed from animal cap cells that were co-treated with activin and angiopoietin-2 (Ang2), expressing the vascular endothelial markers X-msr, Xtie2, and Xegfl7. ${ }^{3,4)}$ In combination with microarray analysis and expression pattern analysis, we identified a novel vascular-expressed gene xrasgrp2 in Xenopus embryos; this gene is a homolog of the human rasgrp2 gene. ${ }^{5)}$ XRASGRP2 over-expressing embryos showed induction of ectopic vascular formation, and its knockdown delayed vascular development. $\left.{ }^{6}\right)$ These results suggest that XRASGRP2 plays a role in vasculogenesis and/or angiogenesis during early development of the Xenopus embryo.

Human RASGRP2, also known as calcium and DAG guanine nucleotide exchange factor I (CalDAG-GEFI), has two major isoforms, which differ with respect to the presence of a 62-amino-acid, amino-terminal extension that encodes a myristoylation/palmitoylation site. The shorter form of RASGRP2, which is located in the cytoplasm, primarily targets Rap1 and Rap2, which are small G-proteins homologous to Ras. ${ }^{7,8)}$

It has been reported that RASGRP2 is involved in leukocyte adhesion deficiency type III (LAD-III) syndrome. ${ }^{9-11)}$ The reports cited above examined hematocytes and molecular mechanisms; however, little is known about the role of this protein in vascular endothelial cells.

The NCBI nucleotide database contains three alternatively spliced transcript variants that encode the same protein from the human rasgrp2 gene; these variants differ in the first exon of the $5^{\prime}$-untranslated region (UTR). Kedra et al. reported that the rasgrp2 gene, which they termed $H C D C 25 \mathrm{~L}$, was located downstream of the pygm gene, by genomic se- quence analysis of a 106-kb region around the pygm gene at $11 \mathrm{q} 13 .{ }^{12)}$

Octamer-binding transcription-factor $1 / \mathrm{POU}$ domain, class 2 , transcription factor 1 (OCT1/POU2F1) is a member of the POU domain family and acts as a transcription factor. Members of this family interact with octamer motifs found in the promoters of many genes that play important roles in immune responses and development. ${ }^{13,14)}$ OCT1/POU2F1 is known to have both activating and silencing activities.

In the present report, we describe the expression profile of rasgrp 2 cDNA in human umbilical artery endothelial cells (HUAECs). We also demonstrate that the $5^{\prime}$-flanking region of the rasgrp2 gene contains a transcriptional repressor-binding region and that OCT1/POU2F1 binds to this region.

\section{MATERIALS AND METHODS}

Cell Culture HUAECs were seeded in 48-well plates $\left(3 \times 10^{4}\right.$ cells per well) in $150 \mu \mathrm{l}$ of EM medium supplemented with growth factors and grown at $37^{\circ} \mathrm{C}$ in a $5 \% \mathrm{CO}_{2}$ atmosphere for $24 \mathrm{~h}$ before transfection.

Reverse Transcription-Polymerase Chain Reaction (RT-PCR) Total RNA was isolated from HUAECs using ISOGEN (Daiichi, Japan), and cDNA was synthesized using random primers and reverse transcriptase. Human cDNA was prepared with mixed RNA from the pancreas, lung, skeletal muscle, brain, kidney, heart, placenta, and liver (Clontech, U.S.A.). For first exon amplification, the rasgrp 2 cDNA was amplified from the HUAEC and human cDNA mix. The forward primers used were as follows: for D1E, 5'-ACCCGCTGCCTGCAGGAAGAC-3'; for M1E, 5'-AGCGCACGGAGGTGGGGTCG-3'; and for P1E, 5'-CGTGGAGAGGCACTGCCTTG- $3^{\prime}$. The reverse primer sequence for all first exons was 5'-ACCTGACCAGGTGGCACGTT-3'.

Vector Construction The DNA fragment of the rasgrp2 promoter region ( $933 \mathrm{bp}$ ) was amplified from human placenta cDNA (Clontech) by using the Expand Long Template kit 
(Roche, Switzerland), digested with HindIII and EcoRI, and cloned into the HindIII/EcoRI sites of the pMetLuc-Reporter vector (Clontech); it was named pLucRasgrp2. The pLucRasgrp2 vector was digested with BamHI and then it was self-ligated; this vector was now named pLuc_B322. Other 131-bp, 147-bp, and 349-bp BamHI fragments were cloned into the BamHI site of the pMetLuc-Reporter vector; the resulting vectors were named pLuc_317/449, pLuc_444/590, and pLuc_585, respectively. The 131-bp, 147-bp, and 349-bp BamHI fragments were cloned into the BamHI site of pLuc_B322; these vectors were named pLuc_B322_317/449, pLuc_B322_444/590, and pLuc_B322_585, respectively. For pLuc_B322_1, pLuc_B322_2, and pLuc_B322_3, sense and antisense oligonucleotides were synthesized and then annealed. These DNA fragments were cloned into the BamHI/ AgeI site of pLuc_B322. The oligonucleotide sequences used were as follows: for pLuc_B322_1, 5'-GATCGGGGGAGTGTTACGGCAGGGAACACTCG-3' and 5'-CCGGCGAGTGTTCCCTGCCGTAACACTCCCCC-3'; for pLuc_B322_2, 5'-GATCAGTCTAGGCCCTCATTATGGGGAAACTGAG$3^{\prime}$ and 5'-CCGGCTCAGTTTCCCCATAATGAGGGCCTAGACT-3'; and for pLuc_B322_3, 5'-GATCCTGAGGTGCGGGGGCGGT-3' and 5'-CCGGACCGCCCCCGCACCTCAG-3'.

The pLuc_B322 vector was digested with SacI and HindIII and then treated with exonuclease III and mung bean nuclease. These clones were named pLuc_B322Del-777, pLuc_B322Del-707, and pLuc_B322Del-665.

The primer set used for the xrasgrp2 promoter region (933 bp) was 5'-CTCAAGCTTACTCCTCTTTGTTGGGA$3^{\prime}$ and 5'-GCAGAATTCGTGGCTATCTGGAGACG-3'.

Luciferase Assay For the luciferase assay, we used the Ready-To-Glow Secreted Luciferase Reporter System (Clontech). HUAECs were transfected with $300 \mathrm{ng}$ of each luciferase vector construct, using Fugene HD (Roche). After $24 \mathrm{~h}, 3 \mu \mathrm{l}$ of substrate was added in $30 \mu \mathrm{l}$ of culture medium, and the luciferase activities were measured with ARVO MX (Perkin Elmer, U.S.A.). Each assay was performed in triplicate, and three independent assays were performed.

Gel Shift Assay Double-stranded DNA was prepared for use as a probe by annealing a biotin-labeled synthetic oligonucleotide, 5'-TCAGTTTCCCCATAATGAGGGCCTAGACT-3', and a non-labeled oligonucleotide, 5'-GATCAGTCTAGGCCCTCATTATGGGGAAACTGAG-3' . Cells were grown in a $10-\mathrm{cm}$ dish and were washed with ice-cold phosphate buffered saline (PBS). Nuclear extracts were isolated using NE-PER Nuclear and Cytoplasmic Extraction Reagents, according to manufacturer's protocol (Pierce, U.S.A.). Finally, nuclear protein was extracted in $100 \mu \mathrm{l}$ of NER. Nuclear extract $(2 \mu \mathrm{l})$ and $22.5 \mathrm{fmol}$ of biotin-labeled DNA were incubated at room temperature in binding buffer containing $10 \%$ glycerol, $5 \mathrm{~mm} \mathrm{MgCl}_{2}, 50 \mathrm{ng} / \mu \mathrm{l}$ poly $(\mathrm{d} I-\mathrm{d} C)$, and $0.05 \%$ NP-40. For gel supershift assays, nuclear extract and an anti-Octl antibody (Santa Cruz, U.S.A.) were incubated at $4{ }^{\circ} \mathrm{C}$ for $30 \mathrm{~min}$ before mixing with binding buffer containing the biotin-labeled DNA. After $20 \mathrm{~min}$ of incubation, the mixtures were analyzed by electrophoresis on a $6 \%$ polyacrylamide gel at $100 \mathrm{~V}$ for $1.5 \mathrm{~h}$ in Novex Tris-Glycine Native Running buffer (Invitrogen, U.S.A.). The gel contents were electrophoretically transferred to a nylon membrane at $380 \mathrm{~mA}$ for $30 \mathrm{~min}$. The membrane was then cross-linked on a transilluminator equipped with $312 \mathrm{~nm}$ bulbs for $15 \mathrm{~min}$. Detection of biotin-labeled DNA by chemiluminescence was conducted using the LightShift Chemiluminescent EMSA kit, according to the manufacturer's protocol (Pierce).

\section{RESULTS AND DISCUSSION}

Rasgrp2 Expression We previously reported that Xenopus rasgrp2, called xrasgrp2, was expressed in vascular endothelial cells during early development. ${ }^{5)}$ In humans, rasgrp 2 was found to be expressed in HUAECs and human umbilical vein endothelial cells (HUVECs), from screening of human endothelial cell lines with RT-PCR. According to the NCBI nucleotide database, there are three alternative variants that differ in the first non-coding exon in the human rasgrp 2 gene.

To investigate the transcripts of the rasgrp 2 mRNA in HUAECs, we carried out RT-PCR analyses using specific primers for each first exon as a forward primer and a common reverse primer derived from the second exon (Fig. 1). This analysis showed that the rasgrp 2 mRNA used only the distal first exon (D1E), and not the middle first exon (M1E) or the proximal first exon (P1E). These results suggest that the upstream region of $\mathrm{D} 1 \mathrm{E}$ is functional in rasgrp 2 transcription in HUAECs. In HUVECs, the mRNA also only used the D1E; this suggests that the rasgrp 2 mRNA containing the D1E was transcribed in vascular endothelial cells (unpublished results).

On the other hand, all transcripts were found in cDNA derived from a tissue mixture containing human pancreas, lung skeletal muscle, brain, kidney, heart, placenta, and liver, and the existence of three transcripts was consistent with the information in the NCBI database. Moreover, we confirmed the expression of all three transcripts in a lymphoblastoid cell line (unpublished results). The use of different promoters may play a role in the differential temporal and spatial expression in tissues and cells.

Promoter Activity of the rasgrp2 Gene As a step towards understanding the molecular mechanisms of rasgrp2 gene expression, we analyzed the promoter activity of the $5^{\prime}$ flanking region of the rasgrp 2 mRNA, starting from D1E, using a luciferase reporter assay in HUAECs. Because the $3^{\prime}$ end of the pygm gene is located in $933 \mathrm{bp}$ upstream of rasgrp, ${ }^{12)}$ it is believed that the promoter activity of the

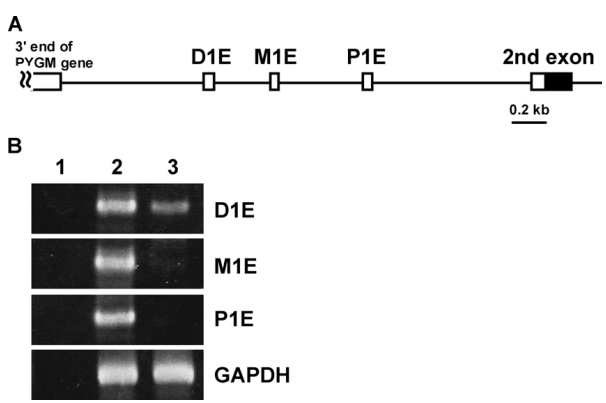

Fig. 1. Analysis of the rasgrp 2 mRNA Expression Using the First Exon

(A) Structure of the 5 '-flanking region of the human rasgrp 2 gene. Open and closed boxes show untranslated and coding regions, respectively. D1E, M1E, and P1E represent the mean distal first exon, middle first exon, and proximal first exon, respectively. (B) RT-PCR analysis of rasgrp2 mRNA in HUAECs and mixed human tissues. GAPDH is an internal control. Lane 1, no DNA; lane 2, a mixture of human tissues; lane 3 , HUAECs. 
rasgrp 2 gene would be within this 933-bp region (Fig. 1).

To define the rasgrp 2 gene promoter, we created BamHIdigested constructs of the 5 '-flanking region and fused them to the luciferase reporter gene. The luciferase activity in HUAECs after transient transfection of the constructs was regarded as an indicator of promoter activity (Fig. 2). The construct pLuc_B322, containing the sequence from nt -933 to -612 , showed the highest promoter activity, suggesting that the promoter activity in the $5^{\prime}$-flanking region of the rasgrp2 mRNA starting from D1E resided in the region from nt -933 to -612 . On the other hand, the construct pLuc_444/590, containing the sequence from $\mathrm{nt}-485$ to -344 , had reduced promoter activity (Fig. 2A).

To confirm whether this region (nt -485 to -344$)$ actu- ally acted as a transcriptional silencer or simply had no transcriptional activity, each BamHI-digested fragment was fused to pLuc_B322 (Fig. 2B). This experiment demonstrated that the construct pLuc_B322_444/590, containing the sequence from nt -485 to -344 , dramatically reduced the promoter activity of pLuc_B322, suggesting the presence of a silencer. The pLuc_B322_317/449 and pLuc_B322_585 sequences also reduced the promoter activity of pLuc_B322, but these activities were higher than that of the construct pLucRasgrp2, which contained the entire sequence of the $5^{\prime}$-flanking region. Additionally, the promoter activities of the constructs pLuc_317/449 and pLuc_585 were about the same as that of pLucRasgrp2 (Fig. 2A). This finding suggests that DNA structural changes, such as loop formation because of the

A
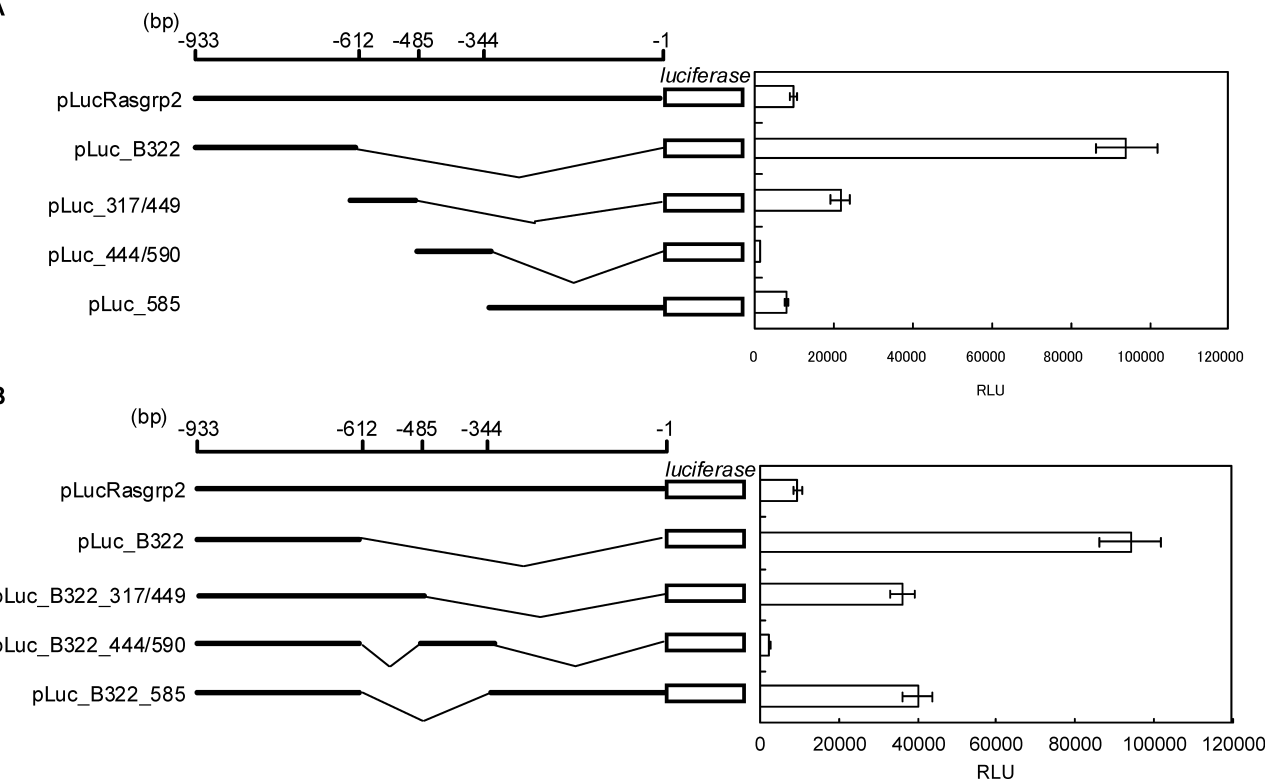

Fig. 2. Promoter Activity of the 5'-Flanking Region of the rasgrp2 Gene

The constructs contained the region between nt -933 and -1 of the $5^{\prime}$-flanking sequence, and the luciferase activity of each construct was examined. (A) Clones constructed from the fragment digested with BamHI. (B) Clones constructed from pLuc_B322 that was fused with each BamHI fragment. The bars represent standard deviations of at least 3 independent experiments. RLU indicates relative light unit.

\section{A $\quad-485$} GGATCCAAAGTCGGGGGAGTGTTACGGCAGGGAACACTCGCAGACCGCC TAGTCTAGGCCCTCATTATGGGGAAACTGAGGAACAGAAAGGTCCTGTT TCTAAGTCTTACATTACCAAGACTGAGGTGCGGGGGCGGTCCTGGATCC

B

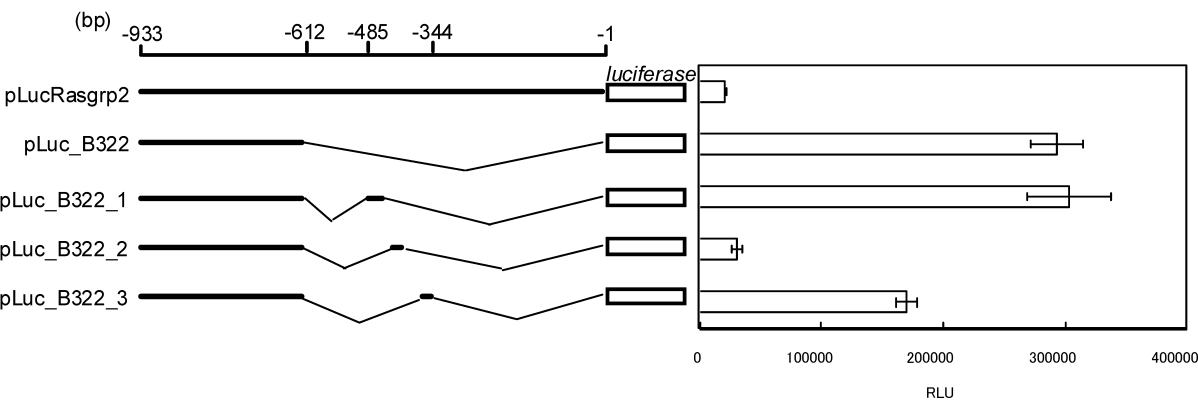

Fig. 3. Promoter Activity of the $5^{\prime}$-Flanking Region of the rasgrp 2 Gene

(A) Sequence between $n t-485$ and -344 . The underlined sequences are those that the TFSEARCH program predicted as having high scores, and regions 1 , 2 , and 3 were predicted to be recognized by HSF2, OCT1, and AML1a, respectively. Bold indicates the OCT1 transcription factor binding site. (B) Clones constructed from pLuc_B322 that was fused with region 1,2 , or 3 . The bars represent standard deviations of at least three independent experiments. RLU indicates relative light unit. 
GC-rich sequences in this region, may have caused the reduction in promoter activity in pLuc_B322_317/449 and pLuc_B322_585. However, the presence of a silencer is also possible.

The results for two constructs, pLuc_444/590 and pLuc_B322_444/590, suggest the presence of a silencer in the region from $n t-485$ to -344 . Thus, this region was our next focus. An analysis using the computer program TFSEARCH ${ }^{15}$ ) predicted the presence of several protein-DNA binding sites in this region. Because three small regions (regions $1-3)$ covered all the identified DNA-binding sites, each sequence was fused with pLuc_B322 (Fig. 3A). The luciferase assay showed that the constructs pLuc_B322_2 and pLuc_B322_3, containing regions 2 and 3, respectively, reduced the promoter activity of pLuc_B322 (Fig. 3B). In particular, construct pLuc_B322_2, containing region 2, markedly reduced the activity; this reduction was comparable with that of pLucRasgrp2. This result suggested the presence of a silencer in region 2. According to the TFSEARCH program, an OCT1/POU2F1-binding motif (CCCTCATTATGG) was present in region 2 .

Identification of a Silencer-Binding Protein To confirm whether nuclear protein(s) are bound to the sequence of region 2, we carried out gel shift assays, using biotin-labeled region 2 DNA as a probe and nuclear extracts prepared from HUAECs. This assay revealed a shift in the DNA-protein complex band (Fig. 4A). To assess the specificity of the shifted band, non-labeled region 2 DNA was added to the reaction mixture. The shifted band decreased by the presence of the competitor, indicating that the nuclear protein specifically recognized the sequence of region 2 .

The TFSEARCH program predicted the presence of an OCT1/POU2F1-binding motif in the sequence of region 2. We next carried out gel super shift assay using an anti-OCT1 antibody. This result revealed that the shifted band disappeared, by blocking the DNA-protein complex, suggesting that OCT1/POU2F1 acts as a transcriptional repressor in the 5 '-flanking region of the rasgrp2 gene. It has been reported that OCT1/POU2F1 can repress the expression of various genes, including the gonadotropin-releasing hormone receptor, growth hormone, and prolactin genes. ${ }^{16-18)}$ Thus,
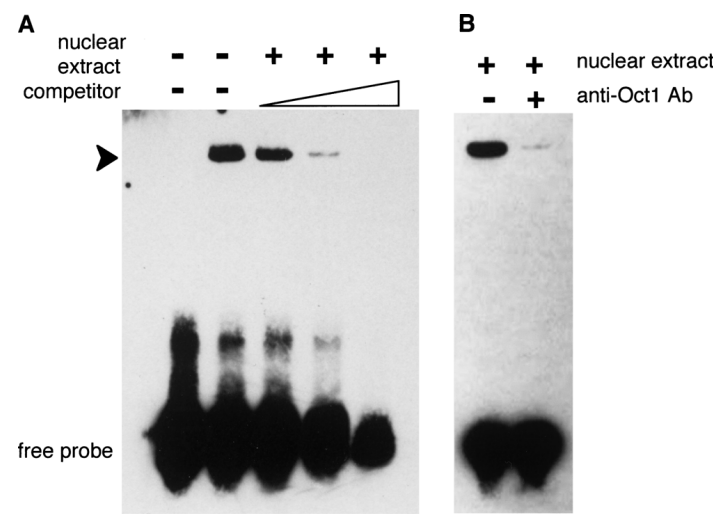

Fig. 4. Gel Shift Assay of the Silencer of the rasgrp2 Gene

(A) Specificity of the DNA-protein complex was tested by competitive analysis. (B) Gel super shift assay using an anti-OCT1 antibody.

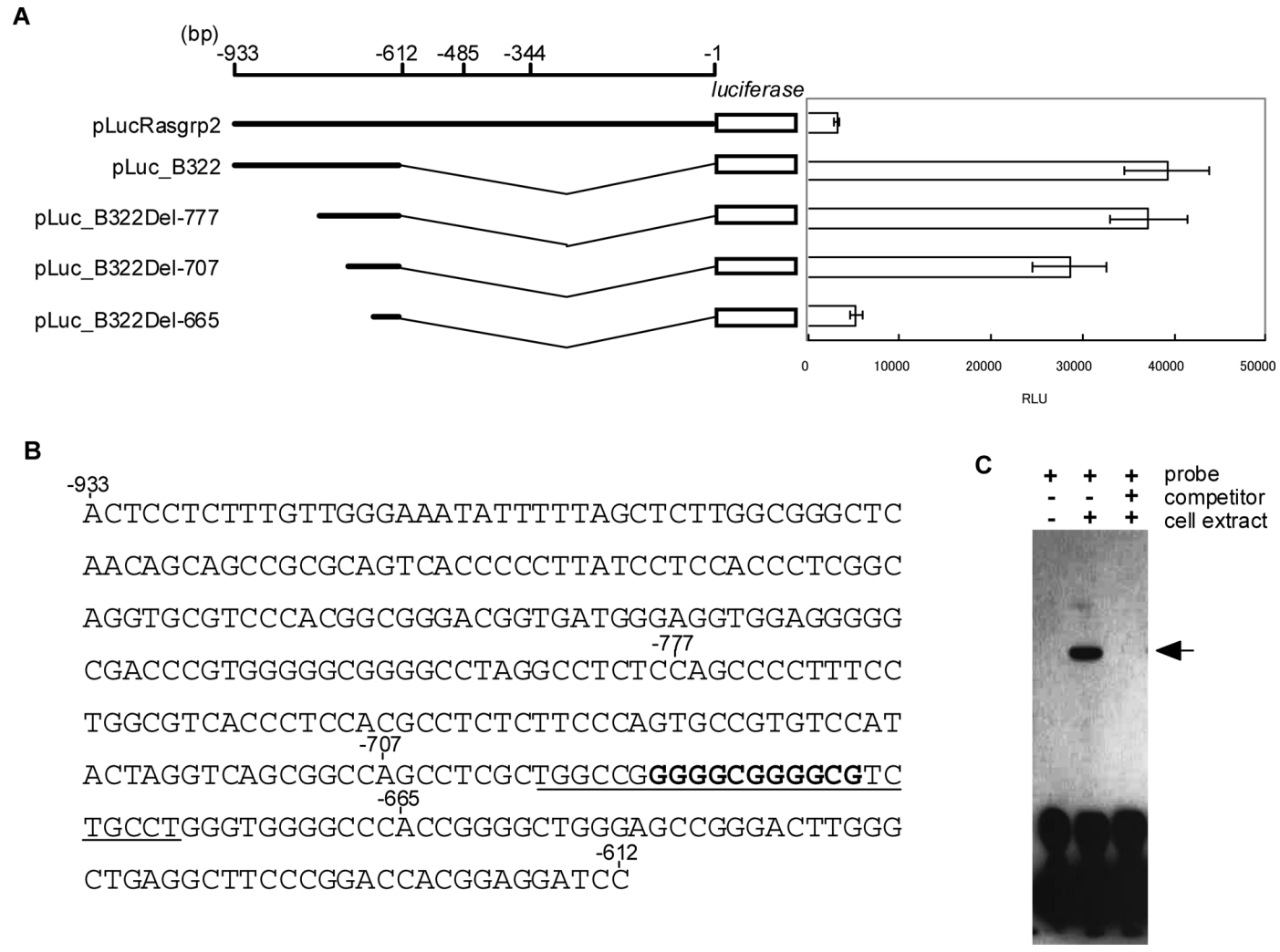

Fig. 5. Promoter Activity of the 5'-Flanking Region of the rasgrp2 Gene

(A) The constructs contained the region between nt -933 and -665 of the 5 '-flanking sequence, and the luciferase activity of each construct was examined. The bars represent standard deviations of at least three independent experiments. RLU indicates relative light unit. (B) The sequence of the region between nt -933 and -665 . Bold indicates the SP1 transcription factor binding site. The underlined elements represent the probe used in the gel shift assay. (C) Gel shift assay of the promoter of the rasgrp2 gene. Specificity of the DNA-protein complex was tested by competitive analysis. 
rasgrp 2 expression may also be down-regulated by OCT1/ POU2F1. However, the mechanism of repression is not well understood.

Identification of the Transcriptional Activator Region Next, we tried to confirm the presence of the transcriptional activator region by the luciferase reporter assay and gel shift assay. The constructs pLuc_B322 and its deletion series, pLuc_B322Del_777 and pLuc_B322Del_707, maintained the highest promoter activity, while the construct pLuc_B322Del_665 showed reduced promoter activity (Fig. $5 \mathrm{~A})$. This suggested that there was a transcriptional activator region between $n t-707$ and -665 . The TFSEARCH program predicted the presence of an SP1-binding site in this region (Fig. 5B). Thus, we performed gel shift assay using a sequence including an SP1-binding site as a probe. This assay revealed a shifted band of the DNA-protein complex (Fig. 5C). When non-labeled DNA was added to the reaction mixture, the shifted band was decreased by the presence of the competitor, indicating that the nuclear protein specifically recognized the sequence. From the prediction of the TFSEARCH program and the result of the gel shift assay, it seems that SP1 binds to this region and activates the promoter of the human rasgrp2 gene.

\section{CONCLUSION}

We demonstrated that the expression of the human rasgrp2 gene was controlled by a promoter and a silencer, and that the OCT1/POU2F1 transcription factor bound to the silencer region. In the present study, we also used HUVECs, and the results were the same as those obtained using HUAECs (unpublished results), suggesting that this expression mechanism is common in vascular endothelial cells. In Xenopus, XRASGRP2, a homolog of human RASGRP2, is involved in vascular development during embryogenesis. The findings of this study may be helpful in the study of vasculogenesis and/or angiogenesis in endothelial cells.

\section{REFERENCES}

1) Springett G. M., Kawasaki H., Spriggs D. R., Bioessays, 26, 730-738 (2004).

2) Stone J. C., Biochem. Soc. Trans., 34, 858-861 (2006).

3) Nagamine K., Furue M., Fukui A., Asashima M., Zoolog. Sci., 22, $755-761(2005)$

4) Nagamine K., Furue M., Fukui A., Matsuda A., Hori T., Asashima M., Biol. Pharm. Bull., 30, 1856-1859 (2007).

5) Nagamine K., Matsuda A., Asashima M., Hori T., Biochem. Biophys. Res. Commun., 372, 886-891 (2008).

6) Suzuki K., Takahashi S., Haramoto Y., Onuma Y., Nagamine K., Okabayashi K., Hashizume K., Iwanaka T., Asashima M., Int. J. Dev. Biol., 54, 609-508 (2010).

7) Clyde-Smith J., Silins G., Gartside M., Grimmond S., Etheridge M., Apolloni A., Hayward N., Hancock J. F., J. Biol. Chem., 275, 3226032267 (2000).

8) Dupuy A. J., Morgan K., von Lintig F. C., Shen H., Acar H., Hasz D. E., Jenkins N. A., Copeland N. G., Boss G. R., Largaespada D. A., J. Biol. Chem., 276, 11804-11811 (2001).

9) Bergmeier W., Goerge T., Wang H. W., Crittenden J. R., Baldwin A. C., Cifuni S. M., Housman D. E., Graybiel A. M., Wagner D. D., J. Clin. Invest., 117, 1699-1707 (2007).

10) Pasvolsky R., Feigelson S. W., Kilic S. S., Simon A. J., Tal-Lapidot G., Grabovsky V., Crittenden J. R., Amariglio N., Safran M., Graybiel A. M., Rechavi G., Ben-Dor S., Etzioni A., Alon R., J. Exp. Med., 204, 1571-1582 (2007)

11) Svensson L., Howarth K., McDowall A., Patzak I., Evans R., Ussar S., Moser M., Metin A., Fried M., Tomlinson I., Hogg N., Nat. Med., 15, 306-312 (2009).

12) Kedra D., Seroussi E., Fransson I., Trifunovic J., Clark M., Lagercrantz J., Blennow E., Mehlin H., Dumanski J., Hum. Genet., 100, 611-619 (1997).

13) Herr W., Cleary M. A., Genes Dev., 15, 1679-16793 (1995).

14) Rosenfeld M. G., Genes Dev., 5, 897-907 (1991).

15) Heinemeyer T., Wingender E., Reuter I., Hermjakob H., Kel A. E., Kel O. V., Ignatieva E. V., Ananko E. A., Podkolodnaya O. A., Kolpakov F. A., Podkolodny N. L., Kolchanov N. A., Nucleic Acids Res., 26, 362367 (1998).

16) Cheng C. K., Yeung C. M., Hoo R. L., Chow B. K., Leung P. C., Endocrinology, 143, 4693-4701 (2002).

17) Kakizawa T., Miyamoto T., Ichikawa K., Kaneko A., Suzuki S., Hara M., Nagasawa T., Takeda T., Mori J., Kumagai M., Hashizume K., J. Biol. Chem., 274, 19103-19108 (1999).

18) Subramaniam N., Cairns W., Okret S., J. Biol. Chem., 273, 2356723574 (1998) 